\title{
Taking large optical quantum states out of the lab: engaging pupils and the public on quantum photonics sciences
}

Matthew Posner, Paolo Mennea, Paul Gow, Rex H. Bannerman, Sam Berry, et al.

Matthew T. Posner, Paolo L. Mennea, Paul C. Gow, Rex H. S. Bannerman, Sam Berry, Devin H. Smith, James C. Gates, Peter G. R. Smith, "Taking large optical quantum states out of the lab: engaging pupils and the public on quantum photonics sciences," Proc. SPIE 10741, Optics Education and Outreach V, 107410D (14 September 2018); doi: 10.1117/12.2319391

SPIE Event: SPIE Optical Engineering + Applications, 2018, San Diego, California, United States 


\title{
Taking large optical quantum states out of the lab: engaging pupils and the public on quantum photonics sciences
}

\author{
Matthew T. Posner*, Paolo L. Mennea, Paul C. Gow, Rex H. S. Bannerman, Sam Berry, \\ Devin H. Smith, James C. Gates, Peter G. R. Smith \\ Optoelectronics Research Centre, University of Southampton, University Road, Southampton, \\ SO17 1BJ, United Kingdom
}

\begin{abstract}
Novel research-inspired outreach activities allow scientists and members of the public to engage in a conversation, increasing the public's understanding and interest in scientific research. This paper reviews outreach and public engagement initiatives undertaken by researchers from the University of Southampton's Optical Engineering and Quantum Photonics Group during a 5-year research program grant entitled Building Large Optical Quantum States. The activities have been supported by a UK Engineering and Physical Sciences Research Council program grant and institutional, national and international professional organizations. The paper discusses activities and hand-outs that have been developed to increase the visibility and public understanding of integrated-photonics fabrication and testing facilities, including a cleanroom-based process for the design and fabrication of quantum outreach chips. More than 1,000 of these chips have been distributed to children, parents and government officials in public events and the activities have contributed towards the authors' research group receiving the 2017 South East England Physics Network Highly Commended Award for Best Research Group. This paper also discusses the impact of these activities and lessons learned.
\end{abstract}

Keywords: Photonics outreach, Public engagement with Research, Optical engineering, Integrated quantum photonics

\section{INTRODUCTION}

The Optical Engineering and Quantum Photonics group at the University of Southampton's Optoelectronics Research Centre have engaged with thousands of members of the public across a variety of events from 2013 to 2018. The activities have been run in conjunction with a program grant that has driven the development of new activities to engage with the public on the fundamental sciences and technologies enabling quantum science experiments. The activities have contributed towards individuals and the group being recognised through awards in outreach and public engagement with research. This paper discusses the development, delivery and impact of these activities and is organised as follows. Section 2 presents the context in which these outreach education and public engagement activities have taken place. Section 3 focuses on the development of "quantum outreach chips" used to engage with different audiences on optical engineering sciences and their applications to quantum information science. Section 4 offers the reader formats of activities that have been used to engage with school students and the public and the evaluation of the impact of these. The paper concludes by offering future perspectives on methods of engagement that may inspire the reader to implement analogous activities in their institution.

\section{BACKGROUND}

This section outlines the socio-economic context driving novel outreach education and public engagement activities in the United Kingdom (UK) through describing the concepts of Public Engagement with Research and Science Capital. The research framework in which activities have been developed based on optical engineering devices for quantum information sciences is also described.

*mp1g09@alumni.soton.ac.uk, orc.soton.ac.uk; planarphotonics.com

Optics Education and Outreach V, edited by G. Groot Gregory, Proc. of SPIE Vol. 10741, 107410D

(c) 2018 SPIE · CCC code: 0277-786X/18/\$18 · doi: 10.1117/12.2319391 


\subsection{Public Engagement with Research}

Public engagement with research (PER) is defined by the UK's National Coordinating Centre for Public Engagement as "a two-way process, involving interaction and listening, with the goal of generating mutual benefit" ${ }^{1}$. Researchers in the UK now have "a strategic commitment to public engagement" and "are recognized and valued for their involvement with public engagement activities" ${ }^{2}$. As a result, they have a duty to demonstrate 'impact', i.e. to show "a demonstrable contribution that excellent research makes to society and the economy" ${ }^{3}$. The activity must be designed accordingly to measure 'impact' through reach and delivery quality. The purpose of photonics "outreach" has consequently been enriched to become an activity encouraging a two-way dialogue between the public and academics about the university's actual photonics research. Consequently, program grants offered through Research Councils UK are often comprised of a "Pathways to Impact" statement, which, amongst other thing, can be targeted to engagement activities inspired by this research and leverage funds to support this. This clause may be used to provide a strategic roadmap to engaging with the public and informing the professional scientific community to influence national and international science policy in the context of research projects. Potential benefits of engaging the public with research can be fed into more theoretical models, such as Science Capital that is described next.

\subsection{Raising Science Capital}

Science Capital is "a conceptual device for collating various types of economic, social and cultural capital that specifically relate to science" ${ }^{4}$. An individual's Science Capital can be considered as an integrated measure of their science related knowledge, skills and experiences and it has been demonstrated that the amount of Science Capital affects attitudes towards science with high scorers more likely to follow STEM subjects. This concept also provides a tool to assess how people engage with science and the influences that impact on this engagement, such as school, home and family, science learning and experiences gained via the environment and everyday life and events. The dynamic environment found in higher education institutions and the education actions they propose can considerably enrich the public's engagement with STEM subjects. The plethora of proposed outreach events can contribute to the raising of an individual's aspirations and thus their Science Capital through increased exposure to different activities and in addition by providing friends and family with information on opportunities available in STEM careers. This in turn plays a mediating role on the aspirations of children towards careers in science ${ }^{5}$. Thus, engaging with a broader range of audiences is key to outreach and public engagement endeavours. Research-inspired activities in this paper form the backbone of a subject of engagement through public engagement with research supported through research grants, with a specific case study relevant to the authors' research work described next.

\subsection{Building Large Optical Quantum States}

The Optical Engineering and Quantum Photonics group (OEQP) of the Optoelectronics Research Centre (ORC) at the University of Southampton has been actively engaging with the public, communicating its diverse research areas. The group has a strong history of collaborating with partners from the University of Oxford to implement novel quantum sciences experiments through their expertise in the design and fabrication of photonic components. The group was part of a program grant awarded by the UK's Engineering and Physical Sciences Research Council (EPSRC) grant, Building Large Optical Quantum States (BLOQS), with the aim to develop an implementation of a large-scale quantum system using light - a quantum optical network - of sufficient size to access this new territory. Through a strategic commitment of delivering value in the research through Pathways to Impact, and with a desire to contribute to raising Science Capital, the authors, who are members of the OEQP group, have developed ways to develop new activities to engage with the public. These will be described in the following section.

\section{BUILDING BLOQS}

The method for showcasing the expertise in optical engineering for the fabrication of BLOQS outreach chips is presented. A cleanroom process and cost structure are presented to realize these devices, with suggestions for process improvement.

\subsection{From photonics quantum science to outreach chip concept}

The authors' expertise lies in the fabrication of optical integrated circuits that will be used as the building blocks for photonics quantum networks, which is based on significant advancements based on waveguide engineering to enable quantum informations sciences experiments, such as Boson sampling ${ }^{6}$ - see Figure 1 (a). The quantum linear photonics system is comprised of sources, linear circuits and detectors, all of which require integration for the scaling to a more 


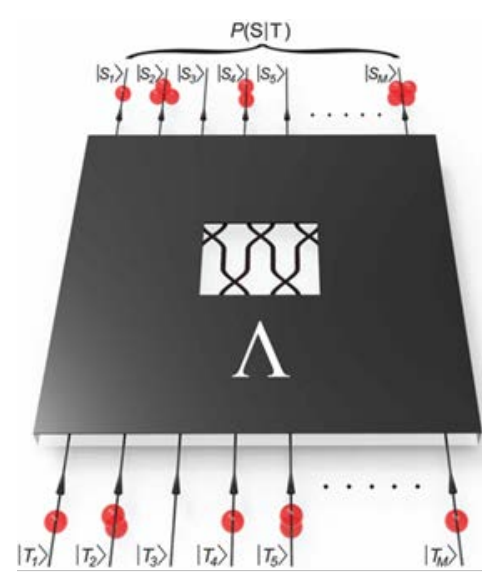

(a)

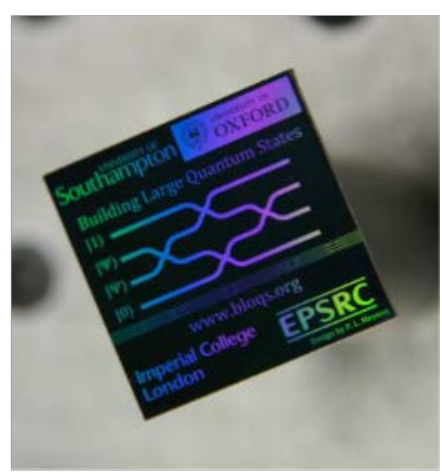

(b)

Figure 1. Mapping concepts for photonics integrated circuits for quantum information processing to devices for the public (a) Implementation of Boson sampling on a photonics integrated circuit. Reprinted from ${ }^{6}$ (b) Quantum chip concept of a Mach-Zehnder interferometer.

complex and novel regime of science. Integration on to a chip necessarily relies on networks of waveguides, used to define the photonics integrated circuits, and other functionalities, such as beam-splitters to form integrated interferometers, or Bragg gratings, for filters for instance. The group's research is built on significant engineering work in cleanroom processes and laser treatment techniques for the definition of these waveguide-circuits. Chips have been designed and realized to provide an insight into the processes used in the research and its application to quantum information science. The circuit on the chips shown in see Figure 1 (b) represent a Mach-Zehnder interferometer, one of the fundamental units for experiments in quantum information processing. The fabrication of these is described next.

\subsection{Process overview}

Silicon wafers (orientation $<100>$, supplier: University Wafers) are used in this process. The wafers are thermally oxidized for in a furnace with a dry oxygen-rich environment at a temperature of 1060C for approximately 12 hours, yielding a thermal oxide of approximately $400 \mathrm{~nm}$ in thickness. The wafers are then processed by photolithography. A positive photoresist (MICROPOSIT ${ }^{\mathrm{TM}}$, S1800 series) is deposited in a 2-step spin process (15 secs at 500 RPM, then 45 secs at $4000 \mathrm{RPM}$ ). The photoresist is baked on a hotplate at 115C for 2 minutes. The resist is exposed using a mask aligner and developed for 50 secs in developer solution (MICROPOSIT ${ }^{\mathrm{TM}}$, MF319). Following the photolithography step, the wafers are etched using hydrofluoric acid. The process is summarized in Figure 2. Further to iterations of the process, it was found that it was possible to remove the resist cleaning after the etching phase, and thus the second spinning of resist to act as a protective coating for dicing.

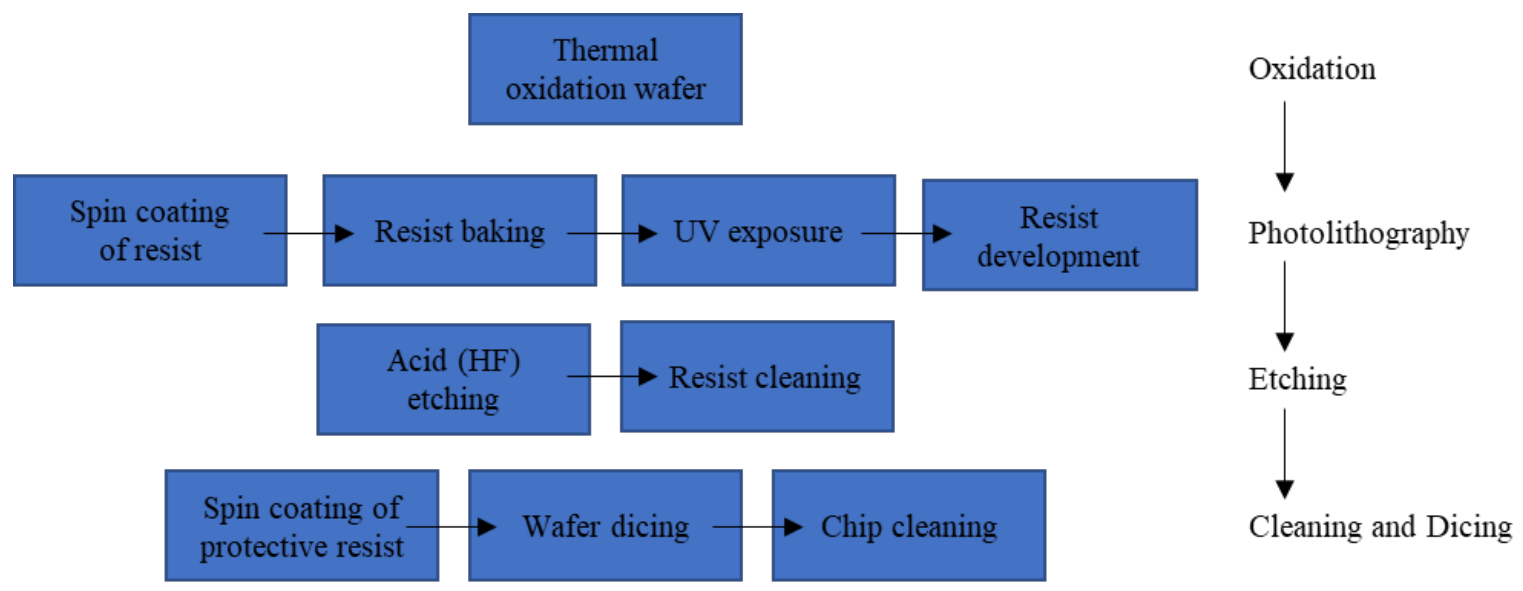

Figure 2. Overview of process flow for chip fabrication. 


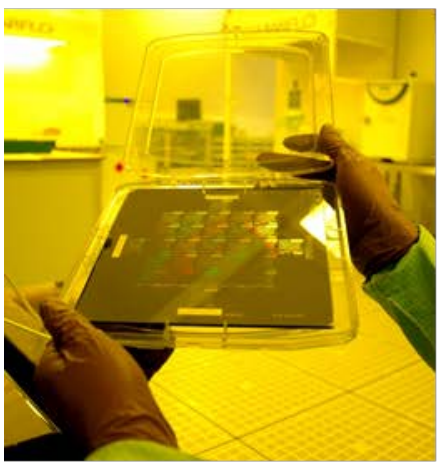

(a)

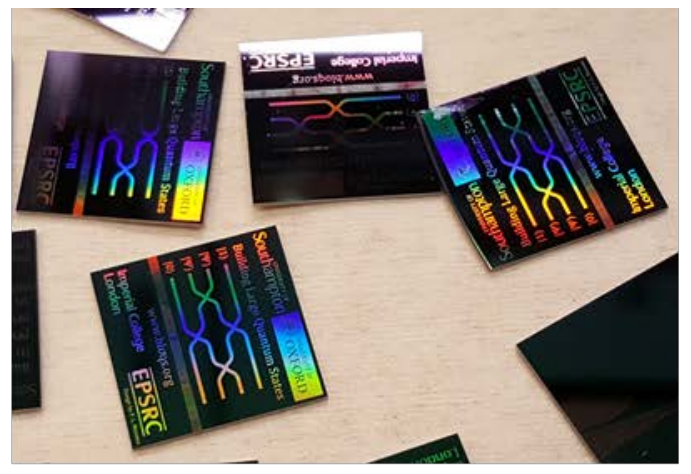

(b)

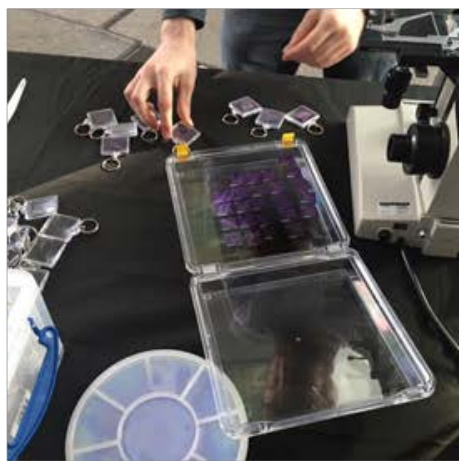

(c)

Figure 3. Fabrication and packaging of BLOQS chips (a) Chromium mask used for photolithography. (b) Diced chips. The periodic etched grating used in the chip caused light diffraction. (c) Packaging of chips into acrylic snap-in key chains .

The associated material costs are the photolithography mask (£35 GBP) - see Figure 3 (a) -, 150 mm diameter wafers (£20 GBP each) and acrylic snap-in key chains (£0.29 GBP each). Each mask can produce 29 chips per wafer. The yield is $80-90 \%$, and the upper range of the yield is obtained through careful handling of the sample and attention to the chips on the edge of the wafer. The cost per chip is $£ 2.38 \mathrm{GBP}$, as calculated with the lower range of the yield. This excludes costs associated with the cleanroom operations and labor, which will vary between facilities. As an indication, a trained operator can produce a batch of 16 wafers, i.e. 400 chips, in up to 3 working days following the process shown in in Figure 2. The chip design is formed of etched gratings, causing light diffraction as seen in Figure 3 (b); this gives an opportunity to explain this fundamental optical concept and relate to the fabrication expertise in Bragg gratings of the OEQP group. The chips can be placed using standard tweezers in the acrylic snap-in keys and looked at under the microscope, providing younger children with a hands-on activity - see Figure 3 (c).

\section{DELIVERY}

The following section provides examples of activities that have been employed during the project to reach different audiences. A summary of the impact achieved is presented as well as lessons learned related to the delivery and evaluation of these outreach education and public engagement initiatives.

\subsection{Formats of activities}

Various types of activities have been employed to engage efficiently with different audiences in both formal and informal environments. Public talks offer a platform to engage with a large audience, generate debate and influence public opinion. The authors have presented at regional, national and international science festivals, with notable features on quantum technology at the 2015 British Science Festival, Pint of Science Festival - see Figure 4 (a) - and the Light Express Laser Show.

The University of Southampton's Lightwave Outreach Education program , led by the lead author for three years during the course of this project, has enabled $\mathrm{PhD}$ students and research staff to obtain training and deliver classes on photonics and technologies to 2,952 students from 81 schools in the South of England ${ }^{7}$. The roadshow has featured run innovative hands-on classes for students to explore the fabrication of photonics integrated circuits - see Figure 4 (b). The quantum chips have also been incorporated alongside portable kits with hands-on experiments with the fundamental sciences underlying this research ${ }^{8}$, and have been presented at international conferences alongside traditional methods for presenting research. Further educational content on the microfabrication engineering techniques could be developed to teach in formal settings for students in high school.

Work in novel environments has also been undertaken. This has permitted the engagement with a wider audience, in particular influencers to the young, to tackle perception towards careers in Photonics and raise Science Capital. The project's research and quantum chips were featured at the 2015 Royal Horticultural Society's Tatton Park Flower Show in the garden "Reflecting Photonics" - Figure 4 (c) - that subsequently won 3 awards, including the South East Physics Network PE Innovation Project Award ${ }^{9}$. 


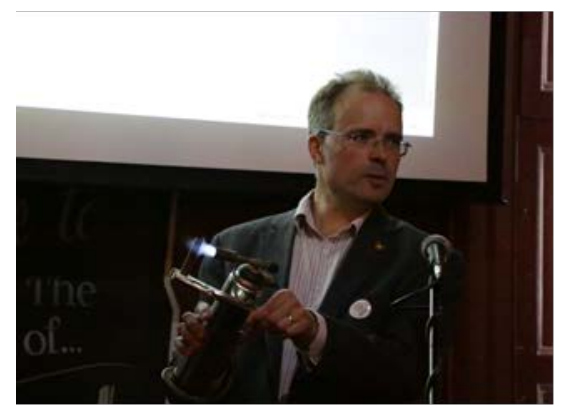

(a)

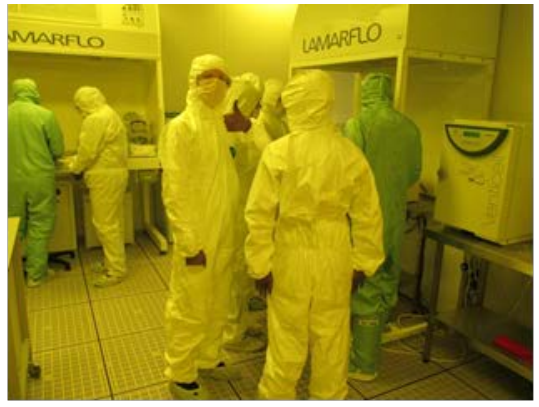

(b)

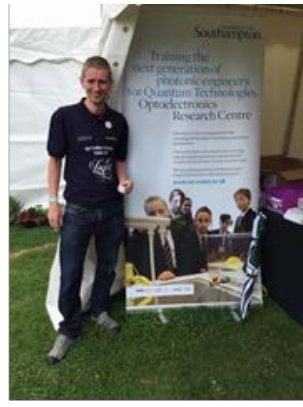

(c)

Figure 4. Activity formats to engage different audiences in informal settings (a) Prof. Peter Smith on non-linear light at the 2015 Pint of Science Festival (b) High-school students from the Godolphin School getting first-hand experience of photolithography in the University of Southampton's Integrated Photonics Cleanroom ${ }^{10}$. (c) Dr. Matthew Posner presenting chips and quantum science at the 2015 Royal Horticultural Society Flower Show Tatton Park.

\subsection{Impact and lessons learned}

In total, 1,000 "quantum chips" have been handed out since the start of the program. The chips are used to mark "lengthy" interactions with members of the public or students, and participants can make their own quantum key ring. The authors have engaged with an estimated 6,000 members of the public and a further 2.952 pupils from 81 schools in the South of England through the formats of delivery described previously. The activities have contributed to the OEQP group receiving the 2017 South East England Physics Network Highly Commended Award for Best Research Group ${ }^{10}$. The varied formats of activities have been used to target different segments of the public and do public engagement in different ways. A recent report published after the completion of these activities will be useful to provide researchers with conceptual frameworks that can be applied to enhance the sophistication of the evaluation ${ }^{11}$. Future activities would benefit from integrating the practical activities into a more theoretical framework.

An essential part of the engagement is to measure the "Impact" in terms of the reach and significance of the activity, and distinguishing general interaction with more significant interaction such as recording significant change of behaviors. Methods will necessarily differ between events, and it is possible to use handouts, online quizzes and surveys can be used. More special items, such as the quantum chips, may be given if it is possible to demonstrate the assimilation of knowledge or change of behavior. Whilst these have been implemented in individual events ${ }^{9}$., a more coordinated approach to obtaining metrics is advised for future work in the context of multi-lateral research collaborations involving multiple institutions. Further research into the implementation of surveys and qualitative assessment for engagement in public events is required. Online resources and training courses are available through the NCCPE to assist in the design and processing of surveys.

\section{CONCLUSION AND FUTURE PERSPECTIVES}

This paper has presented examples of outreach education and public engagement with research activities raising the profile of photonics-enabled quantum science engineering and highlighted some of the measurement tools used to assess the reach and significance of the activities. Examples of Outreach and Public Engagement activities have been present, with the intention of raising the profile of photonics quantum technologies and highlight some of the measurement tools required to assess reach and significance of impact achieved through this activity. In total 1,000 "quantum chips" have been handed the authors have engaged with an estimated 6,000 members of the public and a further 2.952 pupils from 81 schools in the South of England.

\section{ACKNOWLEDGEMENTS}

The authors acknowledge support from the EPSRC-funded program grant Building Large Quantum States out of Light (grant EP/K034480/1); members of the Optical Engineering and Quantum Photonics group and Lightwave Outreach ambassadors from the University of Southampton's Optics and Photonics Society for enthusiasm and participation in supporting these events; Pearl John for guidance and attentive conversation throughout the duration of this project. 


\section{REFERENCES}

[1] "What is Public Engagement?," NCCPE. [Online]. Available: https://www.publicengagement.ac.uk/explore-it/whatpublic-engagement. [Accessed: 06-Aug-2018].

[2] "Concordat for Engaging the Public with Research: RCUK." [Online]. Available:

http://www.rcuk.ac.uk/documents/scisoc/concordatforengagingthepublicwithresearch-pdf/. [Accessed: 06-Aug-2018]

[3] "RCUK Impact Requirements Frequently Asked Questions." [Online]. Available: http://www.rcuk.ac.uk/RCUKprod/assets/documents/impacts/RCUKImpactFAQ.pdf. [Accessed: 06-Aug-2018] .

[4] Archer, L., DeWitt, J., and Willis, B., “Adolescent boys' science aspirations: Masculinity, capital, and power," Journal of Research in Science Teaching 51, 1, (2014)

[5] DeWitt, J. and Archer, L., "Who Aspires to a Science Career? A comparison of survey responses from primary and secondary school students," International Journal of Science Education 37, 2170 (2015).

[6] Spring, J. B., Metcalf, B. J., Humphreys, P. C., Kolthammer, S. W., Jin, X. M., Barbieri, M., Datta, A., ThomasPeter, N., Langford, N. K., Kundys, D., Gates, J. C., Smith, B. J., Smith, P. G. R., and Walmsley, I. A., "Boson sampling on a photonic chip," Science 339, (6121), 798-801 (2013).

[7] Posner, M. T., John, P. V., Wong, N. H. L., Mittal, V., and Nunez-Velazquez, M. M., "From school classes to UNESCO: IYL-enabled environments for tackling the STEM skills shortage through student-led outreach (Invited Paper)," in Proc. SPIE 9946, 9946-6 (2016).

[8] Posner, M. T., John, P. V., Standen, D., Wheeler, N. V., van Putten, L. D., Soper, N., Parsonage, T., Wong, N. H. L., and Brambilla, G., "Reflecting photonics: Reaching new audiences through new partnerships: IYL 2015 and the Royal Horticultural Society flower show," in [Proc. SPIE 9946], 9946-2 (2016).

[9] Wong, N. H. L., Posner, M. T., Mittal, V., Gray, D. R., and John, P. V., "Taking local optics outreach abroad for IYL 2015: administrative and logistical challenges and strategies," in Proc. SPIE 9946, 9946-14 (2016).

[10] "Quantum 'wow'-students look into integrated photonics manufacturing." . [Online]. Available: https://www.orc.soton.ac.uk/news/5117. [Accessed: 06-Aug-2018].

[11] “ORC group recognised in awards ceremony." [Online]. Available: https://www.orc.soton.ac.uk/news/5523 [Accessed: 06-Aug-2018].

[12] Mahony, N. and Stephansen, H. C., "Engaging with the public in public engagement with research," Research for All 1(1), 35-51 (2017). 